\title{
Noninvasive One-Year Ablation Outcome Prediction for Paroxysmal Atrial Fibrillation Using Trajectories of Activation From Body Surface Potential Maps
}

\author{
Yingjing Feng ${ }^{1,2}$, Mirabeau Saha ${ }^{1,2}$, Mélèze Hocini ${ }^{1,2,3}$, Edward Vigmond ${ }^{1,2}$ \\ ${ }^{1}$ IHU Liryc, Electrophysiology and Heart Modeling Institute, fondation Bordeaux Université, \\ Pessac-Bordeaux, France \\ ${ }^{2}$ Univ Bordeaux, IMB, UMR 5251, Talence, France \\ ${ }^{3}$ Bordeaux University Hospital (CHU), Electrophysiology and Ablation Unit, Pessac, France
}

\begin{abstract}
Almost $40 \%$ of paroxysmal atrial fibrillation (AF) patients experience arrhythmia recurrence within a year after initial ablation success. The rich spatiotemporal information provided by body surface potential maps (BSPMs) can reveal AF dynamics. We hypothesised that the dipole direction of the heart during $A F$ can be traced by the centroid trajectory of the principal "activated" electrode patch from the BSPM, where an electrode is defined as "activated" when its signal exhibits a local peak. This hypothesis was first verified using simulated and patient data, indicating that the trajectory has a high correlation with atrial electrical activity. The trajectory was then used as a spatiotemporal feature to predict one-year AF recurrence (22 negative and 23 positive) after ablation among 45 paroxysmal AF patients. The trajectories were segmented according to AF cycles for prediction in a multiple instance classification framework, using a Gaussian mixture regression (GMR) and a linear support vector machine (SVM) with L1 penalty for classification. A leaveone-out test showed 0.73 accuracy, 0.70 sensitivity and 0.77 specificity, and the area under the curve $(A U C)$ of the receiver operating characteristic (ROC) as 0.84 . The work suggests that with the proposed trajectory extracted from the BSPM, the prediction for paroxysmal $A F$ ablation follow-up could be improved.
\end{abstract}

\section{Introduction}

AF is the most common cardiac arrhythmia. For paroxysmal AF, pulmonary vein (PV) isolation performed by radio-frequency catheter ablation (CA) can offer permanent relief. However, the paroxysmal AF has suboptimal midterm and long-term success rates, with almost $40 \%$ of patients experiencing recurrence of $\mathrm{AF}$ within one year [1].

BSPMs refer to electrocardiography signals acquired by multiple leads on the torso. Usually, these leads are ar- ranged to maximally cover the front and the back of the torso, from which rich spatiotemporal information can be extracted. It was sought to determine whether the analysis of these signals could predict whether or not patients remain $\mathrm{AF}$-free after $\mathrm{CA}$ at a one-year horizon. Existing markers to predict immediate $\mathrm{CA}$ success include $\mathrm{AF}$ cycle length (CL); nondipolar component index (NDI), a predictor for termination on persistent AF using BSPM [2]; and spatial variability (SPV), a predictor for short-term ablation outcome on persistent AF using 12-lead ECG [3]. However, none of these methods explicitly takes into account the spatial-temporal movement of the electrical activated region of atria.

This paper presents a method for characterising the spatiotemporal evolution of the heart dipole from preoperative BSPM, and using pattern analysis to classify whether or not the patient remains AF-free one year after CA. Data acquired preoperatively from patients who had undergone CA therapy was used throughout this study.

\section{Methods}

\subsection{Data acquisition and signal processing}

45 paroxysmal AF patients, with one-year recurrence positive (23, with $\mathrm{AF}$ recurrence) or negative (22, remains sinus rhythm (SR) or with only atrial tachycardia (AT)) at $3 \mathrm{M}, 6 \mathrm{M}$, and $12 \mathrm{M}$ follow-ups were obtained from the Bordeaux Cardiology Hospital. Written consent was obtained from all patients. Anti-arrhythmic medications were stopped 48 hours prior to CA. For BSPM acquisition, patients were given diltiazem to slow atrioventricular conduction, and AF was induced with burst pacing. Preoperative BSPMs were obtained through a 252-lead vest (CardioInsight, Medtronic, MN). Vest signals were acquired at a sampling rate of $1024 \mathrm{~Hz} .14 \pm 3$ signals were analysed for each patient, with duration $1020 \pm 236 \mathrm{~ms}$ after QRS-T removal. The CA procedure included PV isolation, 

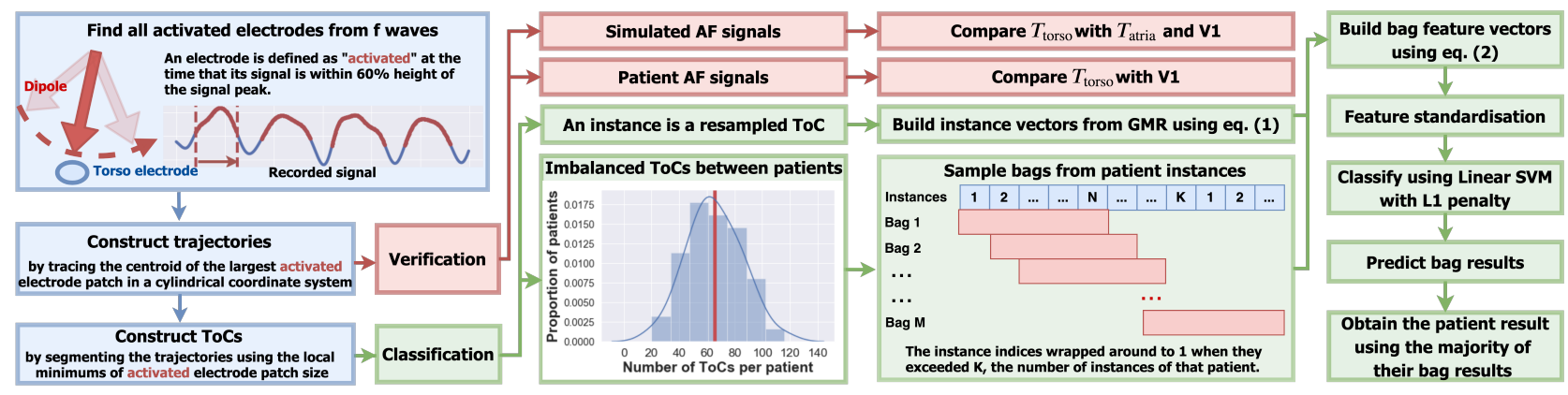

Figure 1: The overall pipeline starting from $\mathrm{f}$ waves to the final recurrence prediction.

and if atria did not convert to SR or AT, sites of phase singularity mapped by catheter, as well as the ECGI solution by CardioInsight, were ablated. For all patients, AF was terminated at the end of procedure.

The QRS segment of the signal was extracted by the Pan-Tompkins algorithm, and the T wave was extracted automatically by a custom method based on the variance of the multi-channel signals. The QRST segments were discarded. The Wilson Central Terminal reference was subtracted from the recorded potentials. A second-order Butterworth filter with a passband of 0.5 to $30 \mathrm{~Hz}$ was applied to remove high-frequency noise and baseline wandering.

\subsection{Trajectory as spatio-temporal feature}

The heart electrical field can be described by a dipole, the orientation of which varies with time. Since the signal of a channel increases when the dipole points at the electrode, the dipole direction can be traced over time by following the path of electrodes exhibiting peaks.

An electrode is defined as activated at the time that its signal is a peak (the first box in Fig 1). To guarantee continuity of the trajectory, all values above $40 \%$ of the height of the local maximum were marked as activated.
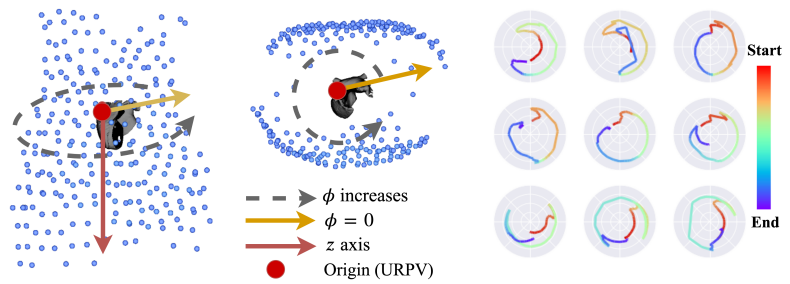

Figure 2: Cylindrical coordinate system of the atria (grey) and vest leads (blue dots) in front (left) and top-down (middle) views. Right: first nine ToCs obtained from the patient signal in Fig 4 in polar plots with radius $z$ and angle $\phi$.

The mean coordinates of the connected activated patch of electrodes at each time step were connected to form a trajectory $T_{\text {torso }}=(\phi(\mathbf{t}), z(\mathbf{t}))$, where $\mathbf{t}$ is the time vector. For arrhythmia with multiple simultaneous dipoles, only the largest continuous patch of activated electrodes was traced to identify the most significant dipole. The positions of the vest electrodes and the atrial nodes were described by $\phi$ and $z$ axes of a cylindrical coordinate system with $(\rho, \phi, z)$, taking the upper right PV (URPV) as origin, and the principal direction of the left atrium on the horizontal plane as $\phi=0$ (Fig 2). Computer simulation and patient data were used to determine the suitability of the torso trajectory to represent the AF activity.
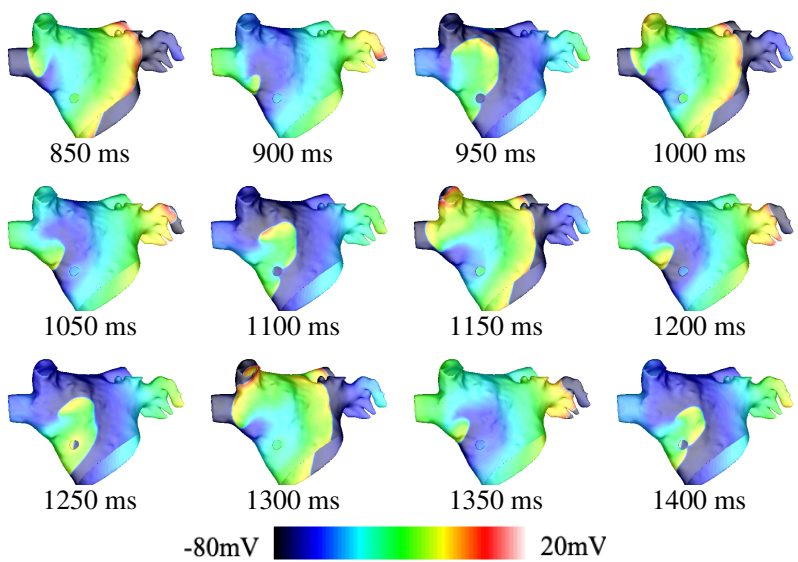

$-80 \mathrm{mV}$ $20 \mathrm{mV}$

Figure 3: Four induced AF re-entries after $850 \mathrm{~ms}$ in the simulated AF. Only the left atrium is shown.

AF reentry was simulated on a biatrial mesh. Vest leads and their relative positions to the atria were derived from a patient scan. Monodomain simulations were performed with the CARPentry simulator [4]. Sinus activation was applied in the model with a $700 \mathrm{~ms}$ cycle length. $220 \mathrm{~ms}$ after the fifth sinus stimulus, five rapid pacing pulses were applied to the URPV with a cycle length $144 \mathrm{~ms}$, modelling PV ectopy to trigger AF. After the last PV stimulus at $796 \mathrm{~ms}$, four reentry cores were seen just under the URPV on the left atrium until $1400 \mathrm{~ms}$, as shown in Fig 3. The extracellular potentials and the local activation time (LAT) of all atrial mesh nodes and 252-channel BSPM were computed, assuming homogeneous torso conductivity. Similar to the previous definition, an atrial node was defined as "activated" under its LATs, and the atrial 
trajectories $T_{\text {atria }}$ were computed by tracing the mean of all activated nodes, and used for comparison with $T_{\text {torso }}$.

For both cases, since the V1 lead of the ECG best reveals atrial depolarisation, its signal was selected to represent the atrial activities. The correlation was assessed by the Pearson correlation coefficient on the frequency spectrum of the standardised data. Over 45 patients, the correlations between $T_{\text {torso }}$ and V1 were $0.75 \pm 0.09$ for $z$ and $0.78 \pm 0.11$ for $\phi$. The high synchronisation can be seen in simulated and real patient examples in Fig 4.
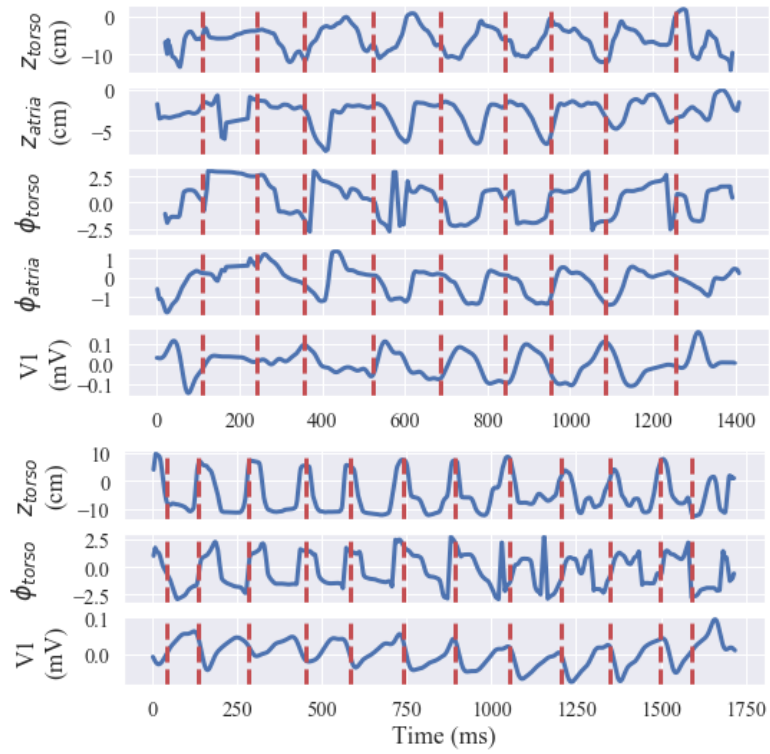

Figure 4: Comparison of $T_{\text {atrial }}, T_{\text {tor so }}$ and the V1 signal, with segmentation shown as red dash lines. Top: from simulation, with high correlations between $T_{\text {torso }}$ and $T_{\text {atrial }}$ ( $z: 0.90, \phi: 0.81$ ), and between $T_{\text {torso }}$ and V1 ( $z: 0.90$, $\phi$ : 0.89). Bottom: from a patient, with high correlations between $T_{\text {tor so }}$ and V1 ( $\left.z: 0.72, \phi: 0.79\right)$.

\subsection{Trajectory classification}

As one signal contains multiple cycles of atrial activity (see Fig 4), segmentation into AF cycles is essential for cycle-to-cycle comparison. Since the activated patch size reflects the strength of the source, its local minimum was used as a marker to segment the trajectory into AF cycles and obtain trajectories of cycles (ToCs), with the constraint that the peak-to-peak interval was $\geq 90 \mathrm{~ms}$ [2] and $\leq 350 \mathrm{~ms}$. A resulting segmentation is shown in Fig 2 and Fig 4. Predicting AF recurrence based on patient ToCs forms a multiple-instance classification problem [5], in which a varying number of ToCs was recorded for each patient were used to predict their one-year clinical outcome.

Each ToC was down-sampled to 20 points, preserving the principal shape while reducing noise. Each re-sampled ToC was defined as an instance $\mathbf{x}_{\mathbf{i}}=\left(p_{1}, p_{2}, \ldots, p_{20}\right)$, and $p_{t}=(\sin \phi(t), \cos \phi(t), z(t), t)$, where we used $(\cos \phi, \sin \phi)$ for continuity near the branch cut of $\phi .4$ instances were randomly resampled four times with placement for each patient, which were selected to be fitted by GMR [6], i.e., all $p_{t}$ of all selected instances were fitted by Gaussian Mixture Model (GMM), to obtain the posterior of $\mathbf{x}_{\mathbf{i}}$ :

$$
\pi\left(\mathbf{x}_{\mathbf{i}} \mid \mathbf{w}_{\mathbf{j}}\right)=\frac{1}{Z_{1}} \sum_{p_{t} \in \mathbf{x}_{\mathbf{i}}} \pi\left(p_{t} \mid \mathbf{w}_{\mathbf{j}}\right)
$$

with $\pi\left(p_{t} \mid \mathbf{w}_{\mathbf{j}}\right)$ being the posterior of $p_{t}$ given the $j^{\text {th }}$ GMM component $\mathbf{w}_{\mathbf{j}}$, and $Z_{1}$ being a normalisation factor s.t. $\sum_{j} p\left(\mathbf{x}_{\mathbf{i}} \mid \mathbf{w}_{\mathbf{j}}\right)=1$. The number of GMM components and the covariance type were selected to have the minimal Bayesian information criterion (BIC), which favoured a high likelihood model while penalising high complexity.

To resolve the problem of imbalanced ToC numbers between patients (Fig 1) and augment training examples, $\mathrm{M}$ bags were gathered for each patient for classification, where each bag contained $\mathrm{N}$ instances of adjacent indices $\mathbf{X}=\left(\mathbf{x}_{\mathbf{k}+\mathbf{1}}, \mathbf{x}_{\mathbf{k}+\mathbf{2}}, \ldots, \mathbf{x}_{\mathbf{k}+\mathbf{N}}\right)$ with $\mathbf{M}$ increasing offsets $\mathbf{k}=0,1, \ldots, \mathbf{M}-1$. The instance indices wrapped around to 1 when they exceeded the number of instances of that patient (Fig 1). Similar to Eq (1), each bag was represented by a feature vector, of which the $j^{t h}$ element was:

$$
v_{j}=\pi\left(\mathbf{X} \mid \mathbf{w}_{\mathbf{j}}\right)=\frac{1}{Z_{2}} \sum_{\mathbf{x}_{\mathbf{i}} \in \mathbf{X}} \pi\left(\mathbf{x}_{\mathbf{i}} \mid \mathbf{w}_{\mathbf{j}}\right)
$$

with $Z_{2}$ being a normalisation factor s.t. $\sum_{j} v_{j}=1$.

Finally, to classify the scarce high-dimensional bag feature vectors resulting from a high number of GMM components, after feature standardisation, a linear SVM classifier with L1 penalty was used to predict the bag results. The percentage of positive bag predictions for each patient was then used as their predictions.

\section{Classification results}

A leave-one-out training-test split was adopted: in each split, 44 out of 45 patients were used for training, and the remaining patient for testing. We compared the effects of varying $\mathrm{M}$ and $\mathrm{N}$ in Fig 5: (a) increased $\mathrm{N}$ and kept $\mathrm{M}=$ 20, AUC-ROC scores were similar, and the optimal results were achieved when $\mathrm{N}=4$ and 5; and (b) increased $\mathrm{M}$ and kept $\mathrm{N}=4$, the AUC-ROC score increased, reached the maximum when $\mathrm{M}=20$, and decreased afterwards, as $\mathrm{M}<$ 20 did not make use of all available instances, and $\mathbf{M}>20$ entailed oversampling from patients with $<\mathrm{M}$ instances. Comparison with other predictors is shown in Fig 5 (c). Table 1 shows the confusion matrix when $\mathrm{M}=20, \mathrm{~N}=4$, and the decision boundary $\geq 0.5$. 


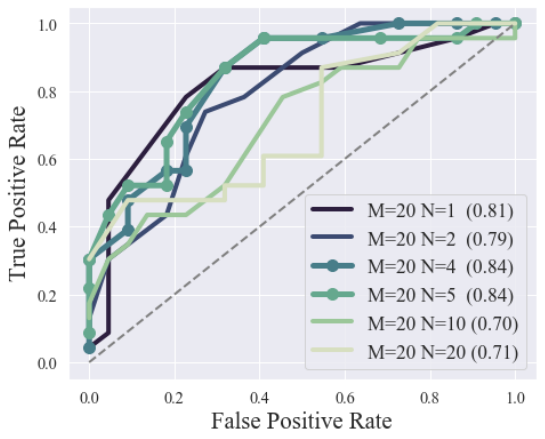

(a)

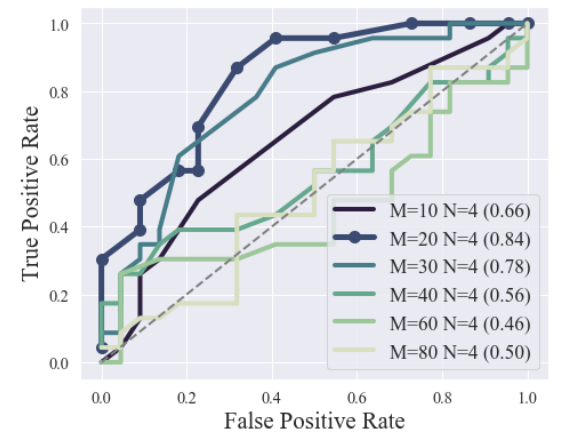

(b)

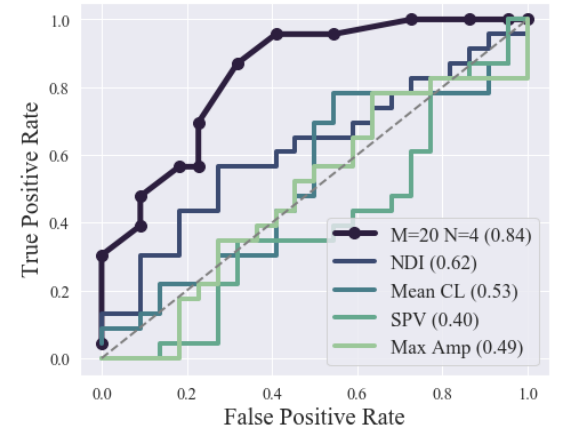

(c)

Figure 5: ROC for prediction with (a)(b) varying $\mathrm{N}$ and $\mathrm{M}$, and (c) comparison with NDI, mean CL of V1, SPV, and the maximum of $\mathrm{f}$ wave amplitude of V1 (Max Amp). The values in brackets are the corresponding AUC-ROC scores.

Table 1: Confusion matrix of patient CA outcome prediction (accuracy: 0.73, sensitivity: 0.70, specificity: 0.77).

\begin{tabular}{|l|c|c|}
\hline & Predicted AF-free & Predicted AF \\
\hline Actual AF-free & $\mathbf{1 7}$ & 5 \\
\hline Actual AF & 7 & $\mathbf{1 6}$ \\
\hline
\end{tabular}

\section{Discussion and conclusion}

In this paper, we hypothesised that the trajectory of activation constructed from the BSPM could characterise the movement of the dipole. The high correlation between proposed trajectories and atrial activities was validated using data both from patients and from computer simulations. Simulations of defined AF dynamics enabled comparing trajectories and the atrial activity, whereas the patient data was used to access the correlation over the population.

Our trajectory-based classification showed high accuracy, sensitivity and specificity in predicting one-year AF recurrence. Our classifier captured the cycle-to-cyle nonstationary dynamics by using ToCs as a spatio-termporal feature and grouping several ToCs for prediction, which explained a better classification result (higher AUC-ROC) compared to other markers, all of which produced only global statistics across many AF cycles. GMR as a generative model for the ToCs, together with BIC for model selection, did not require hyperparameters. Sensitivity analysis of the parameters of the bagging method was studied to reduce the overfitting due to paramter optimization. The best testing score used 20 instances per patient, indicating that $20 \mathrm{AF}$ cycles may be enough for CA outcome prediction for paroxysmal AF.

In conclusion, the hypothesis that the proposed trajectory of activation represents AF dynamics was not rejected, and the trajectory was successfully applied as a spatiotemporal feature to predict one-year CA outcome for paroxysmal AF patients, with a superior result to clinical markers. Future work will focus on translating the findings into clinical practice, such as more aggressive ablation patterns.

\section{Acknowledgments}

This project has received funding from the European Union Horizon 2020 research and Innovation programme "Personalised In-silico Cardiology (PIC)" under the Marie Sklodowska-Curie grant agreement No 764738, the Fondation de la Recherche Médicale (FRM) grant SPF20160936220, and the National Research Agency (ANR) with grant reference ANR-10-IAHU-04. We appreciate Dr. Caroline Roney for providing the bi-atrial mesh.

\section{References}

[1] Ganesan AN, Shipp NJ, Brooks AG, Kuklik P, Lau DH, Lim HS, Sullivan T, Roberts-Thomson KC, Sanders P. Long-term outcomes of catheter ablation of atrial fibrillation: a systematic review and meta-analysis. J Am Heart Assoc 2013;2(2).

[2] Meo M, Pambrun T, Derval N, Dumas-Pomier C, Puyo S, Duchâteau J, Jaïs P, Hocini M, Haïssaguerre M, Dubois R. Noninvasive Assessment of Atrial Fibrillation Complexity in Relation to Ablation Characteristics and Outcome. Front Physiol Jul. 2018;9(JUL):1-19.

[3] Meo M, Zarzoso V, Meste O, Latcu DG, Saoudi N. Spatial variability of the 12-lead surface ECG as a tool for noninvasive prediction of catheter ablation outcome in persistent atrial fibrillation. IEEE Trans Biomed Eng 2013;60(1):2027.

[4] Vigmond EJ, Hughes M, Plank G, Leon L. Computational tools for modeling electrical activity in cardiac tissue. J Electrocardiol Dec. 2003;36(SUPPL.):69-74.

[5] Amores J. Multiple instance classification: Review, taxonomy and comparative study. Artif Intell Aug. 2013;201:81105.

[6] Calinon S, Billard AG. What is the teacher's role in robot programming by demonstration? - toward benchmarks for improved learning. Interact Stud 2007;8(3):441-464(24).

Address for correspondence:

Yingjing Feng

IHU Liryc, F-33600 Pessac-Bordeaux, France

yingjing.feng@ihu-liryc.fr 